\title{
THE
}

\section{Comparative Study of Two Recent Edge-Detection Algorithms Designed to Process Sea-Surface Temperature Fields}

Jean-François Cayula

University of Rhode Island

Peter C. Cornillon

University of Rhode Island, pcornillon@uri.edu

Ronald Holyer

Sara Peckinpaugh

Follow this and additional works at: https://digitalcommons.uri.edu/gsofacpubs

Terms of Use

All rights reserved under copyright.

\section{Citation/Publisher Attribution}

Cayula, J-F., Cornillon, P., Holyer, R., Peckingaugh, S. (1991). Comparative Study of Two Recent EdgeDetection Algorithms Designed to Process Sea-Surface Temperature Fields. IEEE Transactions on Geoscience and Remote Sensing, 29(1), 175-177.

Available at: http://dx.doi.org/10.1109/36.103308

This Article is brought to you for free and open access by the Graduate School of Oceanography at DigitalCommons@URI. It has been accepted for inclusion in Graduate School of Oceanography Faculty Publications by an authorized administrator of DigitalCommons@URI. For more information, please contact digitalcommons-group@uri.edu. 


\title{
Comparative Study of Two Recent Edge-Detection Algorithms Designed to Process Sea-Surface Temperature Fields
}

\author{
Jean-François Cayula \\ Peter Cornillon \\ Ronald Holyer \\ Sara Peckinpaugh
}




\section{Communications}

\section{Comparative Study of Two Recent Edge-Detection Algorithms Designed to Process Sea-Surface Temperature Fields}

\author{
Jean-François Cayula, Peter Cornillon, Ronald Holyer, and \\ Sarah Peckinpaugh
}

\begin{abstract}
Two algorithms used for the detection of fronts in satellitederived sea-surface temperature fields are compared and shown to give similarly good results. The main differences are that the algorithm based on a multilevel approach is faster and that it automatically adapts to process a wide range of SST images and regions within these images.
\end{abstract}

\section{INTRODUCTION}

This correspondence presents a comparative study between two recently developed algorithms [1]-[3] which are designed to automatically detect edges in Sea Surface Temperature (SST) fields. First, an overview of each algorithm is presented. Then the differences between the two methods are highlighted, along with the impact of these differences on the results. For simplicity Algorithm 1 denotes the algorithm designed by Cayula and Cornillon [1], [2], and Algorithm 2, the algorithm developed by Holyer and Peckinpaugh [3].

\section{Overview OF THE Algorithm 1}

Algorithm 1 operates at three levels, much as humans seem to: Picture level, window level, and local/pixel level. Following the input of the data, the most obvious clouds (based on temperature and shape) are identified and tagged so that data which do not represent sea-surface temperature are not used in subsequent modules. These steps operate at the picture and then at the window level. The procedure continues at the window level with the formal portion of the edge detection. Using techniques for unsupervised learning, the temperature distribution (histogram) in each window is analyzed to determine the statistical relevance of each possible front. To remedy the weakness related to the fact that clouds and water masses do not always form compact populations, the algorithm also includes a study of the spatial properties instead of relying entirely on temperatures. In this way temperature fronts are unequivocally defined. Finally, local operators are introduced to complete the contours found by the region-based algorithm. It should be noted that even though local operators are used, they are used in conjunction with the window-based algorithm, and so the qualities of scale invariance and of adaptivity associated with the region-based approach are not lost. As a result, the algorithm takes advantage of both the regional approach and local approach while avoiding their drawbacks. Because portions of Algorithm 1 require smoothed data for optimal performance, and because the overall algorithm is designed to operate on raw input data, these data need to be median-filtered [1]. However, the fields used in [3] and se-

Manuscript received March 7, 1990.

J.-F. Cayula and P. Cornillon are with the Graduate School of Oceanography, University of Rhode Island, Narragansett, RI 02882.

$R$. Holyer and S. Peckinpaugh are with the Computer Science Section, Naval Oceanographic and Atmospheric Research Laboratory, Department of the Navy, Stennis Space Center, MS 39529.

IEEE Log Number 9040157 lected for this study contain a much lower level of noise than that found in the fields normally available at the University of Rhode Island. For this reason the filtering step of Algorithm 1 was not performed for this work.

\section{Overview OF THE Algorithm 2}

Algorithm 2 is a locally based algorithm. By this we mean that each pixel in the image is associated with a measure based on the neighborhood of that pixel. The measure chosen for the algorithm is called the "cluster-shade" and it is computed by using the graylevel co-occurence matrix. The implementation described in [3] uses 16 by 16 pixel windows to compute the gray-level co-occurrence matrix. Edges are identified by detecting zero-crossings within each 3 by 3 pixel neighborhood in the cluster-shade image. Finally, a cleaning/dilation/thinning step can be applied to the edge image. Based on edge morphology, isolated edge pixels are removed and small breaks in edge contours are completed. The resulting contours are one pixel wide. Because this last step modifies contours independently of the SST field, it was not used for the image presented in this correspondence.

\section{Comparison of the Two Algorithms}

The two images selected for this comparative analysis are the images used in [3]. Both images show the SST fields in two areas of the North Atlantic for April 28, 1983.

On the field shown in Fig. 1 the performance of the two algorithms is comparable and most of the noisy area detected by the gradient (Fig. 1(b)) is ignored by both algorithms. Note, however, that finer details (like the edges in the two left-most eddies) are better detected by Algorithm 1 (Fig. 1(d)) than by Algorithm 2 (Fig. 1(c)). Generally, fine edges detected in the gradient image (Fig. 1(b)) are also detected with Algorithm 1 (Fig. 1(d)).

The field shown in Fig. 2 contains significantly more clouds than that shown in Fig. 1 and the SST field is very variable. In this case Algorithm 1 performs very well. For example, the clearly detected edge in the center of Fig. 2(d) is not well defined in Fig. 2(c). The reason for these and other differences in performance is due to the different approaches taken.

The first difference between the two algorithms is that Algorithm 2 operates at the local level only, while Algorithm 1 is a multilevel algorithm. This results in some potential advantage for Algorithm 1. The most obvious advantage is that some features found in SST images can be better identified as a whole rather than at the pixel level. This remark is particularly true when the field is noisy or when clouds are present in the image. A second advantage of Algorithm 1 is that it operates significantly faster than does Algorithm 2 . This results primarily from the fact that Algorithm 1 operates at several levels: At the window level, Algorithm 1 only needs to compute a few complicated statistics (based on simple local statistics) for each window. Then at the local level complicated statistics are computed only to follow and continue contours detected at the window level. This contrasts with Algorithm 2, which evaluates computationally intensive statistics for every pixel in the image. For example, Algorithm 1 processes Fig. 1, a 512 by 512 image, using less than $10 \mathrm{~min}$ of equivalent VAX-11/780 CPU time with more than $50 \%$ of that time used by the cloud-detection portion of the algorithm. On the other hand, the edge-detection portion of Algorithm 2 (without the cleaning/dilation/thinning part) takes about 20 min of equivalent VAX-11/780 CPU time. If a smaller 
(a)

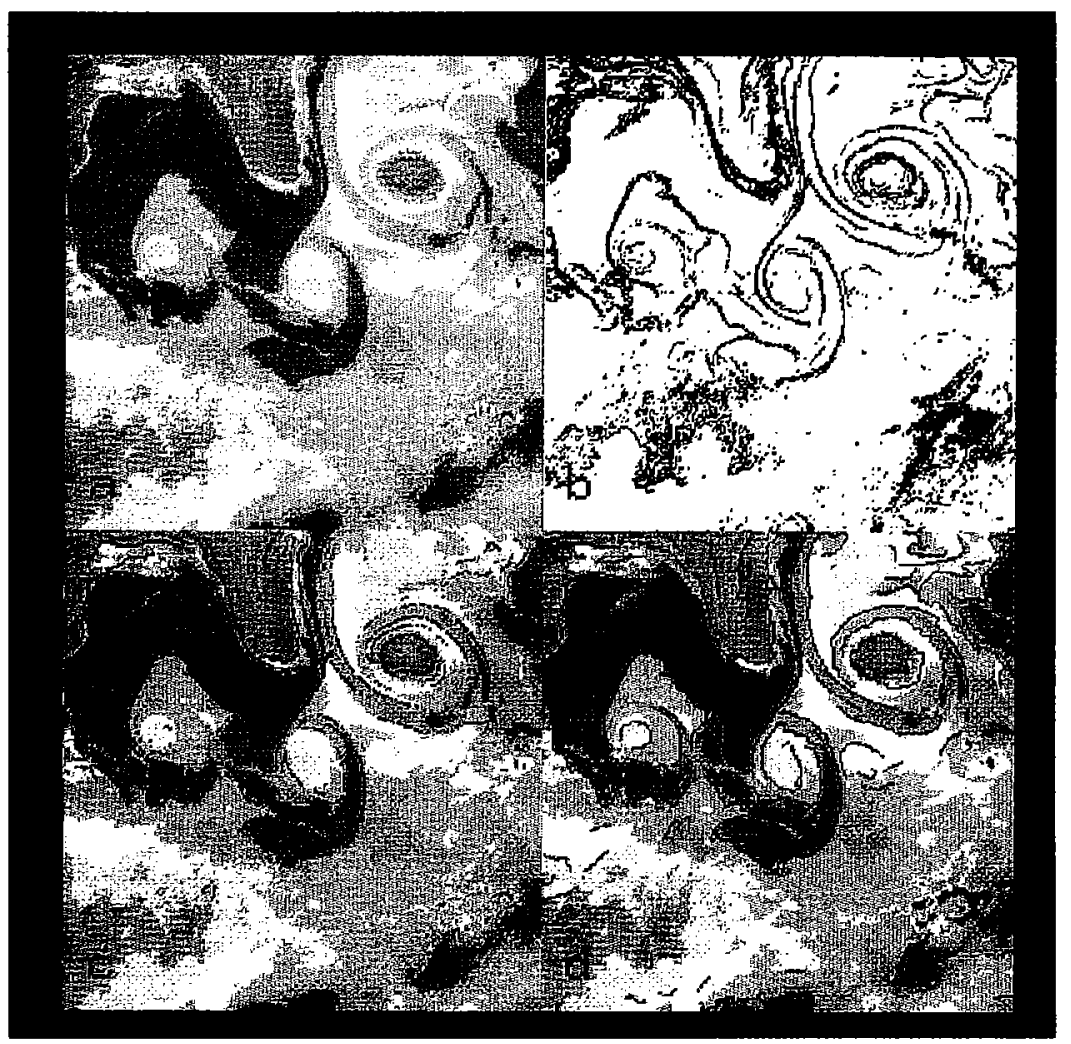

(b)

(d)

Fig. 1. (a) First input image; (b) gradient image threshold at $1^{\circ} \mathrm{C}$; (c) output of algorithm 2; and (d) output of algorithm 1. Detected edges are superimposed in red; detected clouds are shown in black.

(a)

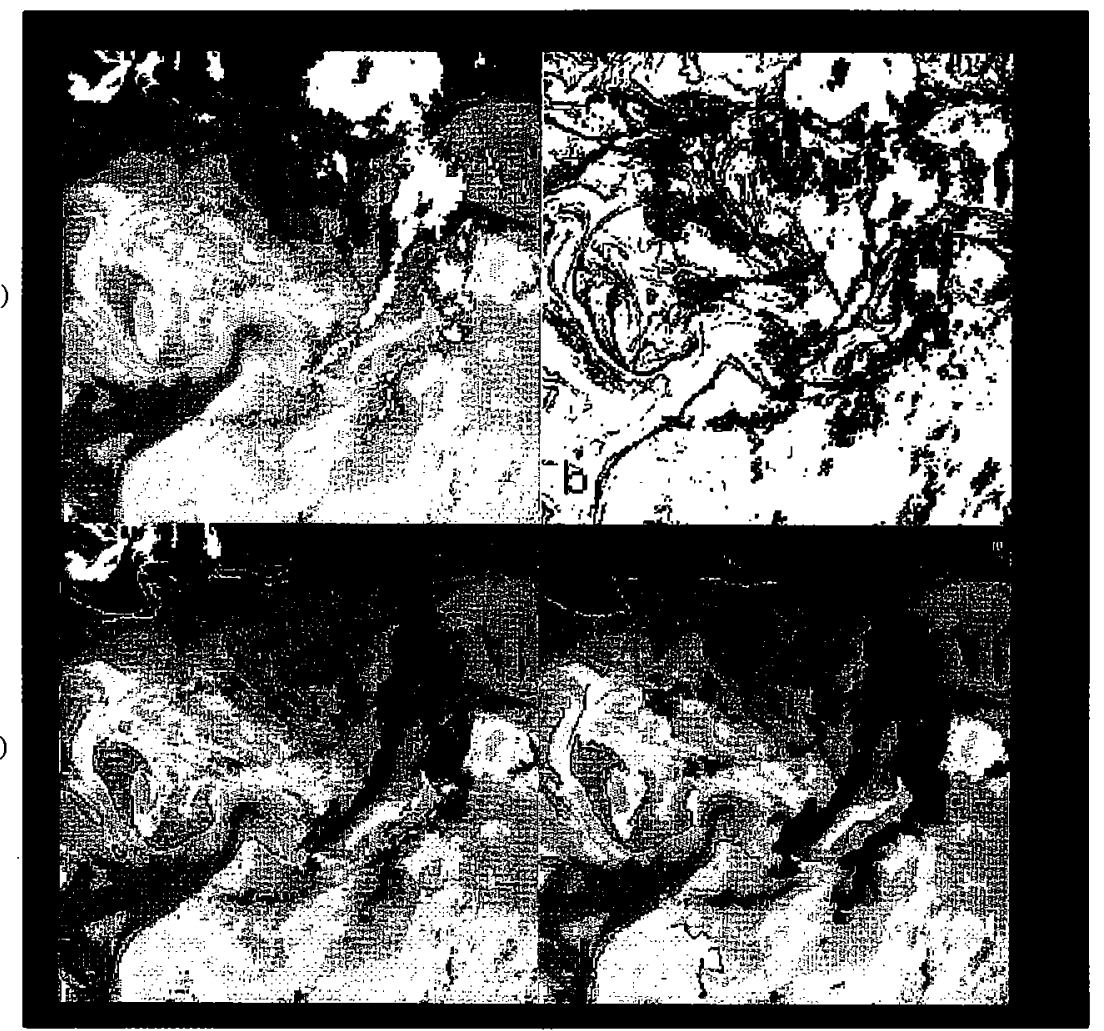

(b)

(d)

Fig. 2. (a) Second input image; (b) gradient image thresholded at $1{ }^{\circ} \mathrm{C}$; (c) output of algorithm 2 ; and (d) output of algorithm 1. Detected edges are superimposed in red or yellow depending on the background; detected clouds are shown in black.

neighborhood was used to compute the cluster shade measure, the execution time of Algorithm 2 could be reduced. However, the cost for this speedup would be less reliable edge detection.

Another difference between the two algorithms is that unlike
Algorithm 2, Algorithm 1 is adaptive and temperature-scale invariant. These two related properties again result from the multilevel approach taken from Algorithm 1 and make the edge detection truly automatic. By this we mean that a temperature threshold does not 
have to be adjusted for each set of images being processed when the temperature scale changes. Furthermore, because the algorithm is adaptive, fine details in low-noise areas of an image can be detected. The automatic nature of Algorithm 1 is probably advantageous in most situations; however, the tunable parameters of Algorithm 2, which give the analyst some control over the degree of smoothing and strength of edges to be detected, could also be an advantage in some cases.

Finally, note that Algorithm 1 includes cloud detection of the SST fields. This is important because analysis done for cloud detection is also used for the frontal detection. Although Algorithm 2 does not include such a cloud-detection portion, it accepts independently generated cloud masks for input. As an example, in [3], a cloud mask was produced using near-infrared data. This example is interesting since it shows that the clouds detected by Algorithm 1 using only data from an SST image (Fig. 2), compares favorably (in this particular case) with the clouds identified by thresholding the near-infrared image corresponding to the SST image.

\section{Conclusion}

In this correspondence we have shown that the two algorithms produced surprisingly comparable results considering the substantial differences in the two approaches: Multilevel (Algorithm 1) versus locally based (Algorithm 2). Algorithm 1 offers the advantage of shorter run times. That advantage is reduced if one is willing to accept a less reliable edge detection by Algorithm 2 . Algorithm 1 also offers the advantage of being adaptive and therefore automatic in its application to different data sets. However, when direct control with regard to detection of the edges is demanded, Algorithm 2 contains two tunable parameters to select the smoothness and the strength of edges, while Algorithm 1 as presently coded does not.

\section{REFERENCES}

[1] J.-F. Cayula, "Edge detection for SST images," M.S. thesis, Dept. Elec. Eng., Univ. Rhode Island, Kingston, 1988.

[2] J.-F. Cayula and P. Cornillon, "Edge detection applied to sea surface temperature fields," in Proc. SPIE Tech. Symp. Optical Eng. and Photonics in Aerospace Sensing, Conf. Digital Image Process. and Visual Commun. Tech. in Earth and Atmos. Sci., 1990.

[3] R. J. Holyer and S. H. Peckinpaugh, "Edge detection applied to satellite imagery of the oceans," IEEE Trans. Geosci. Remote Sensing, vol. 27, pp. 46-56, Jan. 1989. 